\title{
Treatment effectiveness in interstitial cystitis/bladder pain syndrome: Do patient perceptions align with efficacy based guidelines?
}

Avril Lusty; Elizabeth Kavaler; Kay Zakariasen; Victoria Tolls; J. Curtis Nickel

Department of Urology, Queen's University, Kingston, ON, Canada

Cite as: Can Urol Assoc J 2017 Dec. 1; Epub ahead of print. http://dx.doi.org/10.5489/cuaj.4505

Published online December 1, 2017

***

\section{Abstract}

Introduction: We sought to determine if patients' perceptions of success or failure of interstitial cystitis/bladder pain syndrome (IC/BPS) therapies proposed in treatment guidelines align with the evidence from available clinical trial treatment data.

Methods: A total of 1628 adult females with a self-reported diagnosis of IC completed a webbased survey in which patients described their perceived outcomes with the therapies they were exposed to. Previously published literature used in part to develop IC/BPS guidelines provided the clinical trial data outcomes. Patient-reported outcomes were compared to available clinical trial outcomes and published treatment guidelines.

Results: Based on patient perceived outcomes (benefit:risk ratio), the most effective treatments were opioids, phenazopyridine, and alkalizing agents, with amitriptyline and antihistamines reported as moderately effective. The only surgical procedure with any effectiveness was electrocautery of Hunner's lesions. In order of efficacy reported in the literature, the therapies for IC/BPS with predicted superior outcomes should be: cyclosporine A, amitriptyline, hyperbaric oxygen, pentosan polysulfate plus subcutaneous heparin, botulinum toxin A plus hydrodistension, and L-arginine. While some of the guideline recommendations aligned with patient-reported effectiveness data, there was a general disconnect between guidelines and effectiveness reported in clinical practice.

Conclusions: There is a disconnect between real-world patient perceived effectiveness of IC/BPS treatments compared to the efficacy reported from clinical trial data and subsequent guidelines developed from this efficacy data. Optimal therapy must include the best evidence from clinical research, but should also include real-life clinical practice implementation and effectiveness. 


\section{Introduction}

Interstitial cystitis/bladder pain syndrome (IC/BPS) is a urologic pain condition associated with urinary storage symptoms that once diagnosed is managed with a variety of therapeutic strategies. Treatment has generally been empiric but mostly based on theoretical and evidencebased considerations. The development of treatment guidelines, a strategy to improve outcomes by developing a standardized treatment approach, are shaped by efficacy clinical trial data. ${ }^{1-5}$ In real world practice, urologists are encouraged to employ efficacy data and guidelines to prescribe effective therapies and treatments for patients suffering from IC/BPS. There is, however, limited information on the effectiveness of IC/BPS treatments and patients' perceptions of these treatments in real life clinical practice.

The purpose of this study was to determine if treatments IC/BPS patients perceived to be effective in a web-based questionnaire aligned with clinical trial efficacy data and associated published guidelines in IC/BPS.

\section{Methods}

Clinical efficacy considerations were abstracted from a recent comprehensive review/metaanalysis performed by one of the authors (JCN) and colleagues ${ }^{4}$ and guideline updates for the treatment of IC/BPS from the European Association of Urology (EAU), American Urological Association (AUA), Royal College of Obstetricians and Gynaecologists/British Society of Urogynaecologists (RCOG/BSUG) and Canadian Urological Association (CUA). ${ }^{1-4}$ Clinical effectiveness was estimated from a patient centered web-based questionnaire. An initial analysis has been published and describes the diagnostic and therapeutic experience of the first 750 patients completing the survey. ${ }^{6}$ The questionnaire was promoted by IC/BPS support groups and queried the impact of therapies (beneficial and deleterious effects) using a total of 202 unvalidated questions. Standard and common IC/BPS therapies such as oral medications, intravesical therapies and surgery were addressed and patients were asked whether symptoms were improved, deteriorated or were unchanged. The questionnaire was designed and implemented by two of the authors (EK, KZ) and the collected data independently analyzed by the Queen's University group (AL, VT, JCN). Effectiveness was estimated based on benefit:risk ratio (risk being defined as worsening of symptoms) reported by patients (benefit:risk ratio= not effective $=1: 1$ or $<1$; mildly effective $=>1: 1$ but $<5: 1$; moderately effective $=>5: 1$ but $<10: 1$; markedly effective $=>10: 1$ ). Informed consent was obtained online. Data was collected during 2004 to 2010. Descriptive statistics were calculated to compare the efficacy, guideline recommendations and real life effectiveness of the various treatments.

\section{Results}

One thousand six hundred and twenty-eight women with a self-reported diagnosis of IC/BPS responded to the survey. Women reported from 48 different countries with a mean age of 39.6 years (SD+/- 12.8 years). 95.9\% were Caucasian, 1.4\% were African American, and 0.6\% were Asian/Pacific Islander.

Therapies achieving an improvement rate of over 50\% included opioids (405/621; 65.2\%), phenazopyridine (390/638; 61.1\%), intravesical heparin (50/89; 56.2\%), alkalinizing agents (365/660; 55.3\%), and pentosan polysulfate (359/698; 51.4\%). However, in order of effectiveness (based on benefit:risk ratio), marked effectiveness was only achieved with opioids (27:1), alkalinizing agents (22.8:1), and phenazopyridine (13.9:1). Moderate effectiveness was reported for amitriptyline (9.2:1) and antihistamines (6.5:1). Cimetidine, intravesical heparin, 
and intravesical hyaluronic acid were associated with mild effectiveness while electrocautery, pentosan polysulfate, intravesical lidocaine and DMSO showed mild but equivocal effectiveness. Hydrodistension and antibiotics were not effective (although antibiotics were associated with a $49.4 \%$ improvement rate, they also had worsening of symptoms, reported by $47.1 \%$ of patients). Gabapentinoids, major surgery, and urethral dilation were not effective. Table 1 describes the number of patients exposed to each treatment, the improvement and worsening of symptoms reported and the subsequent benefit:risk ratio for each reported treatment modality.

Table 2 summarizes the interventions proven to be efficacious based on clinical trial data (the data was based on pain, frequency, urgency and ICSI symptoms). Table 2 also summarizes the recommendations (and grade of recommendation) for the EAU, AUA, RCOG/BSUG and CUA guidelines compared to patient perceived effectiveness based on the benefit:risk ratio determined for each intervention. ${ }^{1,2,3,4}$

\section{Discussion}

The most recent and accepted definition of IC/BPS from the 2014 AUA guidelines states it is "an unpleasant sensation (pain, pressure, discomfort), perceived to be related to the urinary bladder, associated with lower urinary tract symptoms of more than six weeks' duration, in the absence of infection or other identifiable cause". ${ }^{2,4}$ IC/BPS is a disease complex that is diagnosed after the exclusion of an infectious or neoplastic process. ${ }^{2,4}$ In reality, it appears to take a significant amount of time for patients to obtain a diagnosis. ${ }^{4}$ Theoretically, once the diagnosis has been made, a strategy to flow patients through a treatment algorithm, such as those based on the EAU, AUA, RCOG/BSUG or CUA guidelines, should be the optimal way to manage this condition. ${ }^{1,2,3,4}$ This can only be predicted if the evidence based efficacy data mirrors the actual effectiveness seen in the real world. Clinical trial data would predict that the most beneficial therapies would include cyclosporine A, amitriptyline, hyperbaric oxygen, pentosan polysulfate plus subcutaneous heparin, botulinum toxin A plus hydrodistension and L-arginine, in no particular order. ${ }^{4}$ Guideline recommendations from the EAU, AUA, RCOG/BSUG and CUA follow as best they can with the clinical evidence modified by expert opinion. ${ }^{1,2,3,4}$ Our survey of patients' perceptions of what treatments they actually receive and how much benefit (or harm) they experience does not always align well with either the clinical trial efficacy data or the guideline recommendations.

In this study, the terms efficacy and effectiveness are separate entities. Efficacy is defined as the power to produce a desired result and effectiveness is the degree to which a treatment is successful in producing a desired result. ${ }^{7}$ This study clearly shows there is a disconnect between real world patient perceived effectiveness of IC/BPS treatments compared to the efficacy clinical trial data. Patients perceived the following treatments to be effective: opioids, alkalinizing agents, phenazopyridine, amitriptyline, and antihistamines. Intravesical heparin and pentosan polysulfate were associated with reports of high improvement rates but also of worsening of symptoms making them only moderately effective. Antibiotics were associated with almost half of the patients reporting improvement but also half reporting worsening of symptoms and therefore based on our benefit:risk ratio would be considered not effective. Based on benefit and risk, the other treatments examined showed either equivocal results or no effectiveness.

The only therapies that seem to align between this reported effectiveness and published efficacy include amitriptyline (with the strongest alignment), pentosan polysulfate, hydroxyzine and/or combinations. Most of the treatments noted to have the best efficacy from the review of published literature were associated with either equivocal patient perceived effectiveness or these therapies worsened patients' symptoms. When comparing the patient perceived effectiveness to 
the most recent EAU, AUA, RCOG/BSUG and CUA guidelines, hydroxyzine, amitriptyline and pentosan polysulfate were included as grade A recommendations by the EAU and grade $\mathrm{B}$ and $\mathrm{C}$ recommendations by the AUA and CUA. ${ }^{1,2,4}$ The RCOG/BSUG endorsed amitriptyline with a grade $\mathrm{B}$ recommendation however did not advise hydroxyzine or pentosan polysulfate treatments, with grade B and A recommendations against both treatments, respectively. ${ }^{3}$ The intravesical therapies that patients perceived to have equivocal results, including intravesical DMSO, lidocaine and bladder hydrodistension, were listed as grade A and B recommendations in the EAU but grade $\mathrm{C}$ and $\mathrm{D}$ recommendations in the AUA and CUA guidelines ${ }^{1,2,4}$ and ranged from B, C and D recommendations in the RCOG/BSUG guidelines. ${ }^{3}$ This highlights not only the differences between guidelines based on the same available efficacy data but also some disconnect between patient perceived effectiveness and the recommendations that major urological associations promote. The most glaring example of this disconnect is with opioid therapy. It is certainly interesting that opioids are not only one of the most prescribed medications for IC/BPS, but also perceived to be one of the most effective according to patients exposed to this treatment. Opioids are not recommended in the EAU guidelines while the AUA guidelines "suggest" that opioid therapy can be considered for "multimodal pain therapy"; and list 6 essential principles that should be followed if/when a narcotic is prescribed to a patient for pain management. ${ }^{1,2}$ The RCOG/BSUG suggests opioids should be used with caution in patients with long-term chronic pain and early referral to a pain clinic for patients with refractory symptoms while the CUA guidelines do not discuss opioid treatment. ${ }^{3,4}$ Opioid therapy in IC/BPS, which has been very poorly studied, needs to be rigorously evaluated to determine its role for pain management in IC/BPS.

Why is there such a disconnect between efficacy (and guideline recommendations based on efficacy) and effectiveness? Most of the therapies evaluated in large randomized placebo controlled trials are performed on promising approaches that industry hopes to commercialize. These studies have very rigid inclusion and exclusion criteria that typically enrol only a very small percentage of patients who are screened. The efficacy data may be appropriate in the population studied but may not reflect the actual patient population in clinical practice. Those treatments that are deemed successful, are sometimes financially out of reach for the average patient. Similarly, some treatments recommended in the guidelines (e.g. neuromodulation) are not in general use or available in the real world. The therapies most often used by clinicians are usually generic and funding for evaluating such treatments is almost non-existent. Some of the best approaches include conservative management such as diet manipulation ${ }^{8}$, exercise ${ }^{9}$, improving coping skills and even cognitive behavioural therapy ${ }^{9}$; all treatments never rigorously evaluated. One recent exception is pelvic floor physiotherapy in which the benefits suggested by early small uncontrolled studies have now been confirmed by NIH randomized controlled trials. ${ }^{10,11}$ Physiotherapy is now being prescribed more frequently for IC/BPS patients with pelvic floor dysfunctional pain and we would like to believe that a contemporary survey would show a correlation between patients' perceived effectiveness and clinical trial efficacy. ${ }^{10,11}$

We should and cannot abandon our evidence based approach to developing treatment algorithms and guidelines. We certainly cannot develop these strategies based on uncontrolled data collection of patients' perceptions, however, to be truly effective, there must be some alignment between what we determine in our limited clinical trials and what the patients are actually telling us. We must incorporate patient input of what they value in terms of treatments and outcomes in future treatment algorithm development. To accomplish this, we must try and 
collect real life clinical data in this difficult condition using validated real life clinical practice methodology including prospective observational and/or registry trials.

The limitations to this study are fairly obvious. We are comparing apples (efficacy) and oranges (effectiveness) with the guidelines attempting to provide a balance between the two (expert opinion). The web-based questionnaire included questions that were never validated, and was based on patient self-identified diagnosis, with all the sampling bias that this carries with it (including selection bias and lack of control patients). We were unable to provide clinical confirmation regarding diagnosis, clinical phenotyping (e.g. Hunner's lesion), treatment actually prescribed or therapeutic response but rather relied on self-diagnosis and patients' perception of response. Some of the results are difficult to interpret as few patients received some treatments listed, for example, botulinum toxin A and cyclosporine A, resulting in a small sample size. The questionnaire is dated and may influence this disconnect between patient perceptions and the more recent guidelines, but would not overly influence the comparison to efficacy since most data presented was available at the time of the questionnaire. We also have no information as to how the various treatments were applied in clinical practice (e.g. duration or order of treatment modalities in individual patients). However, real life clinical practice in this enigmatic condition is messy and does not follow the niceties of recommended algorithms. We believe that this questionnaire, with its acknowledged limitations, reflects the IC/BPS patients' real life experience with the treatments we as clinicians regularly administer.

\section{Conclusion}

There is a disconnect between IC/BPS treatment guidelines based on clinical trial data efficacy and effectiveness in real world clinical practice. Optimal therapy must include the best evidence from clinical trial research but should also include patient experience from real life practice. 


\section{References}

1. Fall M, Baranowski AP, Elneil S, et al. EAU guidelines on chronic pelvic pain. Eur Urol 2010:57;35-48.

2. Hanno PM, Erickson DA, Moldwin RM, et al. Diagnosis and treatment of interstitial cystitis/bladder pain syndrome: AUA guideline amendment. J Urol 2015:193;1545-2553.

3. Tirlapur SA, Birch JV, Carberry CL, et al. Management of bladder pain syndrome. BJOG 2016:124;e46-e72.

4. Cox A, Golda N, Nadeau G, et al. CUA guideline: Diagnosis and treatment of interstitial cystitis/ bladder pain syndrome. Can Urol Assoc J 2016:10;136-155.

5. Giannantoni A, Bini V, Dmochowski R, et al. Contemporary management of the painful bladder: A systematic review. Eur Urol 2012:61;29-53.

6. Hill JR, Isom-Batz G, Panagopoulos G, et al. Patient perceived outcomes of treatments used for interstitial cystitis. Urology 2007:7;62-6.

7. Merriam-Webster. Accessed March 22, 2017. http://www.merriam-webster.com/efficacy.

8. Friedlander JI, Shorter B, Moldwin RM. Diet and its role in interstitial cystitis/bladder pain syndrome (IC/BPS) and comorbid conditions. BJU Int 2012:109;1584-1591.

9. Whitmore KE. Complementary and alternative therapies as treatment approaches for interstitial cystitis. Rev Urol 2002:4;S28-35.

10. FitzGerald MP, Anderson RU, Potts J, et al. Randomized multicenter feasibility trial of myofascial physical therapy for the treatment of urological chronic pelvic pain syndromes. J Urol 2013:189;S75-85.

11. FitzGerald MP, Payne CK, Lukacz ES, et al. Randomized multicenter clinical trial of myofascial physical therapy in women with interstitial cystitis/painful bladder syndrome and pelvic floor tenderness. J Urol 2012:187;2113-8. 
Figures and Tables

\begin{tabular}{|l|c|c|c|}
\hline Table 1. Patient-reported treatment effectiveness & Worse & Benefit:risk ratio \\
\hline Treatment & Improved & & $27: 1$ \\
\hline Opioids & $65.5 \%(405 / 621)$ & $2.4 \%(15 / 621)$ & $22.8: 1$ \\
\hline Alkalinizing agents & $55.3 \%(365 / 660)$ & $2.4 \%(16 / 660)$ & $13.9: 1$ \\
\hline Phenazopyridine & $61.1 \%(390 / 638)$ & $4.4 \%(28 / 638)$ & $9.2: 1$ \\
\hline Amitriptyline & $45.5 \%(231 / 512)$ & $4.9 \%(25 / 512)$ & $6.5: 1$ \\
\hline Antihistamines & $35.1 \%(203 / 579)$ & $5.4 \%(31 / 579)$ & $3.2: 1$ \\
\hline Cimetidine & $27.6 \%(54 / 196)$ & $8.7 \%(17 / 196)$ & $2.9: 1$ \\
\hline Intravesical heparin & $56.2 \%(50 / 89)$ & $19.1 \%(17 / 89)$ & $2.2: 1$ \\
\hline Intravesical hyaluronic acid & $31.9 \%(22 / 69)$ & $14.5 \%(10 / 69)$ & $1.6: 1$ \\
\hline Electrocautery & $26.4 \%(31 / 121)$ & $16.5 \%(20 / 121)$ & $1.5: 1$ \\
\hline Pentosan Polysulfate & $51.4 \%(359 / 698)$ & $34.5 \%(241 / 698)$ & $1.2: 1$ \\
\hline Intravesical lidocaine & $33.3 \%(6 / 18)$ & $27.8 \%(5 / 18)$ & $1: 1$ \\
\hline Antibiotics & $49.4 \%(674 / 1364)$ & $47.1 \%(642 / 1364)-\mathrm{N}$ & $1.1: 1$ \\
\hline Intravesical DMSO & $36.9 \%(148 / 401)$ & $32.7 \%(131 / 401)$ & $1: 1$ \\
\hline Hydrodistension & $27.9 \%(218 / 780)$ & $27.6(215 / 780)$ & $0.6: 1$ \\
\hline Gabapentanoids & $26.1 \%(58 / 222)$ & $4.6 \%(99 / 222)$ & $0.3: 1$ \\
\hline Major surgery & $14.3 \%(28 / 196)$ & $41.3 \%(81 / 196)$ & $0.1: 1$ \\
\hline Urethral dilation & $22.5 \%(115 / 512)$ & $22.3 \%(114 / 512)$ & $\mathrm{N}$ not sufficient \\
\hline BoNT/A intravesical injection & $\mathrm{N}$ not sufficient & $\mathrm{N} \mathrm{not} \mathrm{sufficient}$ & \\
\hline
\end{tabular}




\begin{tabular}{|c|c|c|c|c|c|c|}
\hline $\begin{array}{l}\text { IC/BPS treatment } \\
\text { recommendations }\end{array}$ & Efficacy $^{*}$ (effect) & $\begin{array}{l}\text { EAU grade of } \\
\text { recommendation }\end{array}$ & $\begin{array}{c}\text { AUA } \\
\text { Recommendation } \\
\text { - grade and use } \\
\end{array}$ & $\begin{array}{c}\text { RCOG/BSUG } \\
\text { grade of } \\
\text { recommendation }\end{array}$ & $\begin{array}{l}\text { CUA grade of } \\
\text { recommendation }\end{array}$ & Effectiveness $^{* *}$ \\
\hline Opioids & ID & NR & $\begin{array}{c}\text { Should be } \\
\text { initiated with a } \\
\text { multimodal } \\
\text { therapy regimen }\end{array}$ & $\mathrm{NR}$ & NR & $\begin{array}{l}\text { Markedly } \\
\text { effective }\end{array}$ \\
\hline Alkalinizing agents & ID & NR & $\mathrm{NR}$ & NR & NR & $\begin{array}{l}\text { Markedly } \\
\text { effective }\end{array}$ \\
\hline Phenazopyridine & ID & NR & NR & NR & NR & $\begin{array}{l}\text { Markedly } \\
\text { effective }\end{array}$ \\
\hline Analgesics & ID & $\mathrm{C}$ & $1^{\text {st }}$ line & Recommended $^{* * *}$ & NR & ID \\
\hline Amitriptyline & $\begin{array}{l}\text { Small-great } \\
\text { (ICSI, pain, } \\
\text { urgency, and } \\
\text { frequency) }\end{array}$ & A & $2^{\text {nd }}$ line; $B$ & $\mathrm{~B}$ & Optional; B & $\begin{array}{l}\text { Moderately } \\
\text { effective }\end{array}$ \\
\hline Cimetidine & $\begin{array}{c}\text { Small (urgency + } \\
\text { frequency) }\end{array}$ & NR & $2^{\text {nd }}$ line; B & B & Optional; B & Mildly effective \\
\hline $\begin{array}{l}\text { Hydroxyzine } \\
(+/-P P S)\end{array}$ & $\begin{array}{c}\text { Small (ICSI + } \\
\text { pain + urgency + } \\
\text { frequency) } \\
\end{array}$ & A & $2^{\text {nd }}$ line; $C$ & $\begin{array}{c}\text { Not } \\
\text { recommended; B }\end{array}$ & $\begin{array}{c}\text { Optional; C (with } \\
\text { allergic } \\
\text { phenotype) } \\
\end{array}$ & ID \\
\hline Intravesical heparin & ID & NR & $2^{\text {nd }}$ line; $C$ & $\mathrm{D}$ & Recommended; C & Mildly effective \\
\hline $\begin{array}{l}\text { Intravesical } \\
\text { hyaluronic acid }\end{array}$ & ID & B & NR & 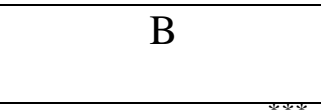 & Optional; C & Mildly effective \\
\hline Electrocautery & ID & $\begin{array}{c}\text { Recommended } \\
\text { (for Hunner's } \\
\text { lesion) } \\
\end{array}$ & $3^{\text {rd }}$ line; $\mathrm{C}$ & Recommended $^{* * *}$ & $\begin{array}{c}\text { Recommended; B } \\
\text { (for Hunner's } \\
\text { lesion) }\end{array}$ & Mildly effective \\
\hline
\end{tabular}


Lusty et al:

Interstitial cystitis/bladder pain syndrome

\begin{tabular}{|c|c|c|c|c|c|c|}
\hline Pentosan polysulfate & Small (pain) & $\mathrm{A}$ & $2^{\text {nd }}$ line; $\mathrm{B}$ & $\begin{array}{c}\text { Not } \\
\text { recommended; A }\end{array}$ & Optional; D & Mildly effective \\
\hline $\begin{array}{l}\text { Intravesical } \\
\text { lidocaine (+ sodium } \\
\text { bicarbonate) }\end{array}$ & $\begin{array}{c}\text { Medium } \\
\text { (urgency); Small } \\
\text { (ICSI + pain + } \\
\text { frequency) }\end{array}$ & NR & $2^{\text {nd }}$ line; $B$ & B & Recommended; B & Mildly effective \\
\hline Antibiotics & $\begin{array}{c}\text { Small } \\
\text { (frequency) }\end{array}$ & NR & NR & $\begin{array}{c}\text { Not } \\
\text { recommended }^{* * *}\end{array}$ & NR & Not effective \\
\hline Intravesical DMSO & ID & A & $2^{\text {nd }}$ line; $C$ & $\mathrm{C}$ & Recommended; B & Not effective \\
\hline Hydrodistension & Small (ICSI) & $\mathrm{C}$ & $3^{\text {rd }}$ line; $\mathrm{C}$ & $\mathrm{D}$ & Optional; C & Not effective \\
\hline Gabapentanoids & ID & NR & NR & NR & $\begin{array}{l}\text { Optional; C (for } \\
\text { neuropathic pain) }\end{array}$ & Not effective \\
\hline Major surgery & Inconclusive & NR & $6^{\text {th }}$ line; $C$ & $\mathrm{D}$ & $\begin{array}{l}\text { Optional; C } \\
\text { (severe } \\
\text { refractory/last } \\
\text { resort) }\end{array}$ & Not effective \\
\hline Urethral dilation & ID & NR & NR & NR & NR & Not effective \\
\hline $\begin{array}{l}\text { Intravesical BoNT/A } \\
\text { (+ hydrodistension) }\end{array}$ & $\begin{array}{c}\text { Great (pain); } \\
\text { Medium (ICSI); } \\
\text { Small } \\
\text { (frequency) }\end{array}$ & NR & $4^{\text {th }}$ line; $\mathrm{C}$ & B & Optional; C & ID \\
\hline Cyclosporine A & $\begin{array}{c}\text { Great (ICSI + } \\
\text { pain }+ \\
\text { frequency) }\end{array}$ & A & $5^{\text {th }}$ line; $\mathrm{C}$ & $\mathrm{D}$ & $\begin{array}{l}\text { Optional; C (last } \\
\text { resort with } \\
\text { inflammation) }\end{array}$ & ID \\
\hline $\begin{array}{l}\text { Intravesical } \\
\text { chondrointin sulfate }\end{array}$ & $\begin{array}{c}\text { Small (ICSI + } \\
\text { pain }+ \\
\text { frequency) }\end{array}$ & B & NR & $\mathrm{D}$ & Optional; D & ID \\
\hline Hyperbaric oxygen & Great (ICSI + & NR & NR & NR & Optional; & ID \\
\hline
\end{tabular}


Lusty et al:

Interstitial cystitis/bladder pain syndrome

\begin{tabular}{|l|c|c|c|c|c|}
\hline & $\begin{array}{c}\text { pain); Medium } \\
\text { (frequency); } \\
\text { Small (urgency) }\end{array}$ & & & refractory to other \\
options
\end{tabular}

${ }^{*}$ Based on size of impact on specific symptoms. ${ }^{* *}$ Benefit:risk ratio=not effective $(1: 1$ or $<1)$; mildly effective $(>1: 1$ but $<5: 1)$; moderately effective $(>5: 1$ but $<10: 1)$; markedly effective $(>10: 1) .{ }^{* * *}$ Best practice recommendation based on the clinical experience of the guidelines development group. ${ }^{* * * *}$ Efficacy dose dependent. ${ }^{* * * * *}$ Great (pain + frequency) when combined with subcutaneous heparin. ID: insufficient data; NR: no recommendation. 